\section{Early-stage diffuse unilateral subacute neuroretinitis: improvement of vision after photocoagulation of the worm}

CA de A Garcia', AHB Gomes', CA de A Garcia Filho' and RNG Vianna ${ }^{2}$
${ }^{1}$ Department of Ophthalmology Federal University of Rio Grande do Norte Natal, Brazil

\section{${ }^{2}$ Department of}

Ophthalmology

Fluminense Federal

University

Niteroi, Brazil

Correspondence: CA de A Garcia

Prontoclinica de Olhos

Rua Ceara Mirim 316

Tirol Natal Rio Grande DO Norte Brazil, CEP: 59020240

Tel: +5511842115888

Fax: +5511842115888

E-mail: prontoc.de.olhos@ digi.com.br

Received: 29 April 2003 Accepted: 27 May 2003 Published online: 9 January 2004

\begin{abstract}
Purpose: To evaluate the visual outcome after direct laser photocoagulation of the worm in patients with early-stage diffuse unilateral subacute neuroretinitis (DUSN).

Methods: We report on four patients with DUSN diagnosed in its early stage, in whom the worm was identified and destroyed by laser photocoagulation.

Results: In all the four cases, resolution of the inflammatory multifocal lesions was achieved within 1 month of treatment. After that period and during follow-up (mean $\mathbf{1 1 . 5}$ months), visual acuity improved in three eyes and remained unchanged in one eye.

Conclusion: The diagnosis of DUSN in its early stage followed by prompt location and destruction of the worm by photocoagulation may improve vision of affected patients. Eye (2004) 18, 624-627. doi:10.1038/sj.eye.6700742 Published online 9 January 2004

Keywords: diffuse unilateral subacute neuroretinitis (DUSN); laser; photocoagulation; visual acuity
\end{abstract}

\section{Introduction}

Diffuse unilateral subacute neuroretinitis (DUSN) is an ocular inflammatory syndrome caused by a single subretinal nematode described initially by Gass et al. ${ }^{1}$ Occurring mainly in children and young adults, it affects one or, rarely, both eyes and usually results in severe loss of vision if left untreated. ${ }^{1,2}$

DUSN has two distinct stages. The early stage is characterized by inflammatory signs such as papilitis, choroiditis, and mild to moderate vitritis, and in the late stage by narrowing vessels, optic nerve pallor, and focal or diffuse changes in the retinal pigment epithelium (RPE). ${ }^{1}$

The diagnosis of the disease in its early stage is important, because identification and destruction of the worm with laser photocoagulation prevents further visual loss and can occasionally lead to improved vision.

This paper reports on four patients with DUSN in its early stage, in whom the worm was identified and destroyed by photocoagulation, leading to regression of inflammatory signs and improved vision in three patients.

\section{Materials and methods}

Medical record review was performed for all patients seen at the Uveitis clinic, Department of Ophthalmology, Federal University of Rio Grande do Norte, Natal, Brazil, between January 2001 and January 2003. A total of 70 patients with DUSN were identified. However, only four patients diagnosed in the early phase of the disease were enrolled in this study. Earlystage DUSN is characterized by mild or moderate vitritis, mild optic disc oedema, and recurrent crops of evanescent, multifocal, greywhite lesions at the level of the outer retina. ${ }^{1}$ The round, often glistening white worm is tapered at both ends and always found in the vicinity of these grey-white lesions.

The four patients with active DUSN underwent Snellen visual acuity testing, slitlamp examination, fundus examination with indirect ophthalmoscopy, and/or a 78-diopter Volk lens. The worm was located in all patients. 
Argon laser treatment was performed under topical anaesthesia via a dilated pupil. The laser beam was focused onto the retina using a Goldman three-mirror contact lens. The spot size ranged from 200 to $300 \mu \mathrm{m}$. Power settings ranged from 150 to $200 \mathrm{~mW}$ with an exposure time of $0.2 \mathrm{~s}$. The worm was covered with confluent laser spots until the exposed area appeared whitish.

Immediately after laser treatment, neither systemic nor topical medications were prescribed. The patients were re-examined $48 \mathrm{~h}$, 1 week, 2 weeks, 1 month, and every 2 months after photocoagulation.

\section{Results}

Clinical data and treatment results are shown in Table 1. The four patients were men with a mean age of 14.2 years (ranging from 9 to 23 years) at onset of symptoms. They all had unilateral disease and all presented vitreous cells, multifocal chorioretinal lesions and papilitis. All fellow eyes were normal. Patients 1 and 3 presented visual acuity of $20 / 25$ in the affected eye; patient 4 had 20/60, and patient 2 had $20 / 400$.

The worm was located in all patients. In patient 1 , it was located superior to the optic disc; in patient 2 , it was temporal to the foveal region (Figure 1a); in patient 3 , the worm was found inferior to the optic disc (Figures 2 and 3 ); and in patient 4, it was inferior to the foveal region.

After laser treatment, none of the patients showed a decrease in visual acuity. However, intraocular inflammation was exacerbated 1 day after treatment in patients 3 (Figure 2a) and 4 . This included $+/ 4$ vitreous cells and periphlebitis in the region surrounding the

Table 1 Clinical data of patients with DUSN

\begin{tabular}{|c|c|c|c|c|c|c|c|c|}
\hline Patient no. & Sex & Age (years) & Symptoms & Symptomatic eye & $\Delta t$ (days) & Initial VA & Follow-up (months) & Final VA \\
\hline 1 & M & 10 & Blurred vision & Right & 15 & $20 / 25$ & 15 & $20 / 20$ \\
\hline 2 & M & 15 & Decreased vision & Left & 91 & $20 / 400$ & 19 & $20 / 200$ \\
\hline 3 & M & 23 & Blurred vision & Left & 14 & $20 / 25$ & 8 & $20 / 20$ \\
\hline 4 & M & 9 & Decreased vision & Right & 75 & $20 / 60$ & 4 & $20 / 60$ \\
\hline
\end{tabular}

$\Delta t=$ time from onset of visual loss to laser photocoagulation.

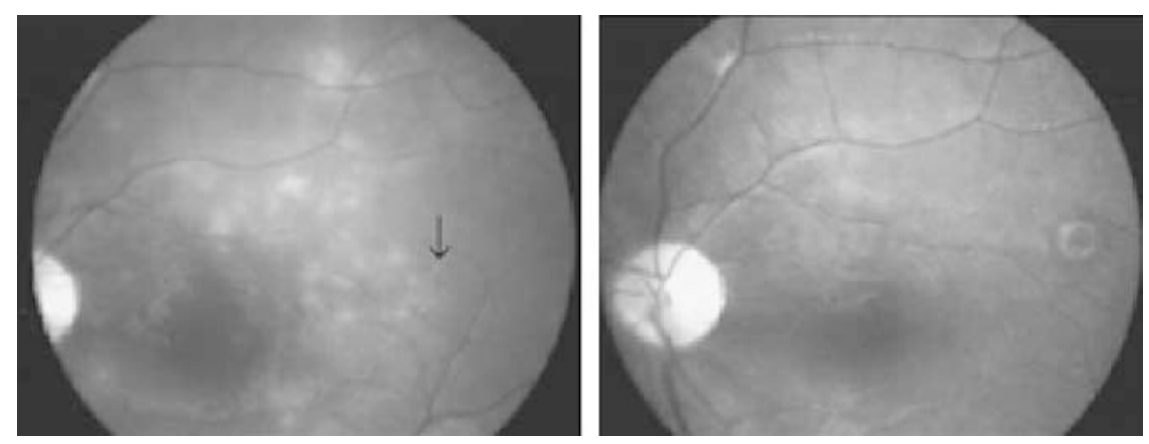

Figure 1 (a) Patient 2. Fundus photograph of the left eye. Observe the multifocal yellow-white choroidal lesions in the posterior pole and the worm located temporal to the fovea (arrow). (b) After 6 months of laser photocoagulation. The fundus appears normal, except for the round, hyperpigmented laser scar temporal to the fovea.
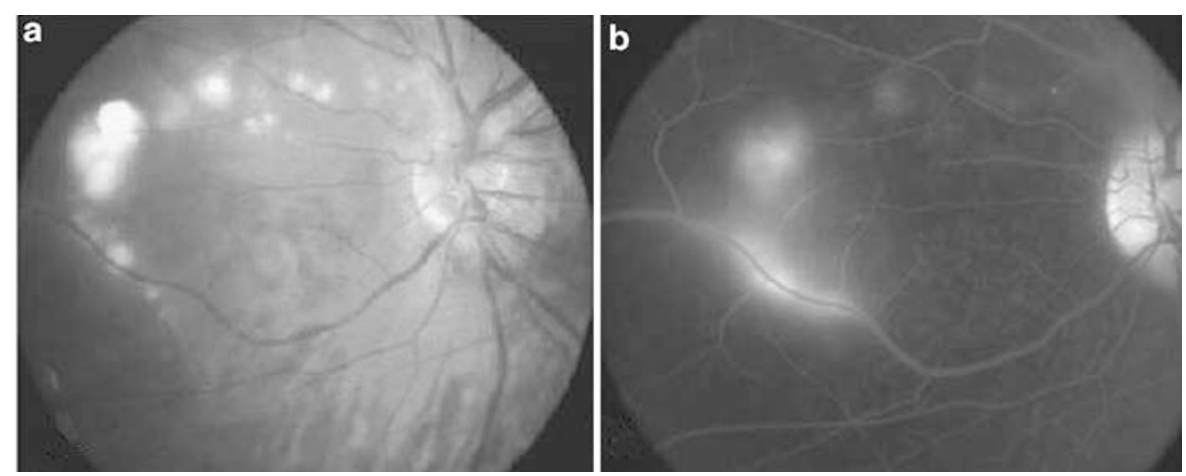

Figure 2 (a) Patient 3. Fundus photograph of the left eye revealing the white-yellowish lesions in the nasal retina and papillitis. (b) Fluorescein angiography reveals perivascular staining and optic disc hyperfluorescence. 
previously photocoagulated area. Although the visual acuity of both patients remained unchanged, patient 3 was treated with oral corticosteroids (prednisone $40 \mathrm{mg}$ / day) for 1 week, after which the inflammation disappeared and the medication was withdrawn. The intraocular inflammation observed in patient 4 resolved within few days without treatment.

After a mean follow-up interval of 11.5 months post-laser (ranging from 4 to 19 months), patients 1 and 3 recovered $20 / 20$ vision, patient 2 had 20/200, and patient 4 had 20/ 60. In all eyes, no signs of intraocular inflammation were seen on slit-lamp examination. Ophthalmoscopy revealed a small, round, hyperpigmented retinal scar located in the previously photocoagulated area of the treated eyes (Figures $1 b$ and $4 b$ ).

\section{Discussion}

DUSN is an infectious disease caused by a nematode which wanders in the subretinal space causing

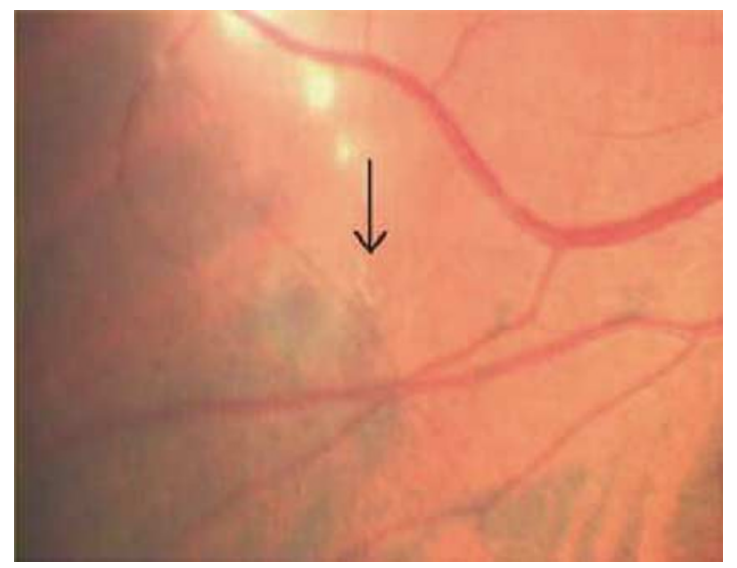

Figure 3 Patient 3. Detail of the left eye revealing the subretinal nematode located inferonasally (arrow). inflammation and degeneration of the posterior retina. This disorder affects otherwise healthy children or young adults and often causes profound loss of vision in one eye. ${ }^{1}$ The pathogenesis of DUSN appears to involve a local toxic tissue effect on the outer retina caused by the worm by-products left in its wake, as well as a more diffuse toxic reaction affecting both inner and outer retinal tissues. ${ }^{1}$ Thus, the loss of visual function is explained on a pathophysiologic rather than on anatomic basis. This may explain the significant loss of visual acuity in patient $2(20 / 200)$ despite the almost normal appearance of the fundus after laser treatment (Figure 1b).

It has been postulated that the diagnosis of DUSN in its early stages and subsequent location and destruction of the worm by photocoagulation may prevent the loss of vision in affected eyes. ${ }^{1}$ Unfortunately, most patients in South America come to be examined in very late stages of the disease, presenting ophthalmoscopic features such as optic atrophy, narrowing of the retinal arteries, focal and diffuse degenerative changes in the RPE and neurosensory retina, and severe permanent loss of vision. In our series of 70 patients with DUSN from an endemic area in Northeastern Brazil, only four were diagnosed in the early stage of the disease. Besides the high degree of suspicion required to diagnose early DUSN, other factors may delay treatment. For example, locating the worm in the retina can be a tedious and time-consuming task requiring many visits.

Another complicating factor is that DUSN may mimic toxoplasmosis, tuberculosis, or syphilis, diseases commonly seen in South America. It is not unusual to find patients with DUSN being treated mistakenly for these diseases.

Although children rarely complain of unilateral loss of vision, a reality in which specialists must contend, patients 1 and 4 were seen by their ophthalmologists because of their ocular complaints, and were
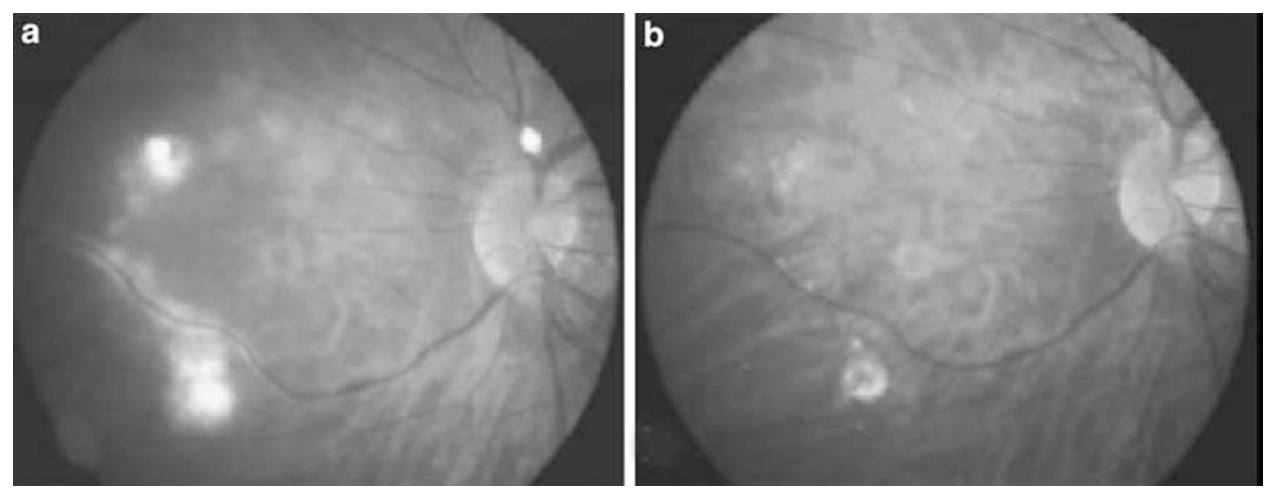

Figure 4 (a) Patient 3. Fundus photograph revealing exacerbation of inflammation 2 days after laser treatment. (b) Fundus photograph after 8 months of follow up. Observe the inferonasally hyperpigmented scar secondary to photocoagulation. The optic disc appears normal. 
immediately referred to our Uveitis Clinic to be evaluated.

Several cases of DUSN have been reported presenting a visual outcome following direct photocoagulation of the worm. ${ }^{3-8}$ However, most of these patients were treated in the late stage. $3,5,7,8$

In the present paper, we report four cases in which DUSN was diagnosed in its early stage. After a mean follow-up of 11.5 months, visual acuity improved in three patients and remained stable in one after laser treatment. This supports Gass' observation that visual acuity often does not improve after photocoagulation unless the worm is killed soon after onset of visual loss. ${ }^{1}$ This was clearly observed in our series. Patients 1 and 3 had a shorter delay ( 2 weeks) between the onset of visual symptoms than patients 2 and 4 ( 2 month or more) and experienced better visual outcomes. Gass believes that the visual improvement observed after photocoagulation of the worm results from clearing of the vitreous humor cellular infiltrate and reduction in optic nerve oedema. ${ }^{1}$

Direct photocoagulation of the worm exacerbated inflammation in patients 3 and 4, probably due to liberation of the nematode's cell wall antigen. The inflammatory process was well controlled with shortterm steroid therapy in patient 3. The intraocular inflammation seen in patient 4 resolved without any medication.

Thus, in cases of unilateral multifocal chorioretinitis, DUSN should be suspected, especially if patients come from endemic areas. Early diagnosis and adequate treatment are paramount to saving vision in affected eyes.

\section{References}

1 Gass JDM. Diffuse unilateral subacute neuroretinitis. In: Gass JDM Stereoscopic Atlas of Macular Diseases; Diagnosis and Treatment, 4th edn. CV Mosby: St Louis, 1997, pp 622-628.

2 Cunha de Souza E, Abujamra S, Nakashima Y, Gass JDM. Diffuse unilateral subacute neuroretinitis: first patient with documented nematodes in both eyes. Arch Ophthalmol 1999; 117: 1349-1351.

3 Raymond LA, Gutierrez Y, Strong LE, Wander AH, Buten R. Iving retinal nematode (filarial-like) destroyed with photocoagulation. Ophthalmology 1978; 85: 944-949.

4 Gass JDM, Braunstein RA. Further observations concerning the diffuse unilateral subacute neuroretinitis syndrone. Arch Ophthalmol 1983; 101: 1689-1697.

5 Carney MD, Combs JL. Diffuse unilateral subacute neuroretinitis. Br J Ophthalmol 1991; 75: 633-635.

6 Sivalingam A, Goldberg RE, Augsburger J, Frank P. Diffuse unilateral subacute neuroretinitis. Arch Ophthalmol 1991; 109: 1028 .

7 Goldberg MA, Kazacos KR, Boyce WM, Everett AI, Katz B. Diffuse unilateral subacute neuroretinitis. Ophthalmology 1993; 100: 1695-1701.

8 Moraes LR, Cialdini AP, Avila MP, Elsner AE. Identifying live nematodes in diffuse unilateral subacute neuroretinitis by using the scanning laser ophthalmoscope. Arch Ophthalmol 2002; 120: 135-138. 